\title{
On the Syntactic Structure of Chinese Ambiguity Sentences
}

\author{
Weiwei Dai \\ Southwest Minzu University, Chengdu, China \\ Email: 7289209@qq.com
}

How to cite this paper: Dai, W.W. (2021) On the Syntactic Structure of Chinese Ambiguity Sentences. Open Access Library Journal, 8: e7917.

https://doi.org/10.4236/oalib.1107917

Received: September 1, 2021

Accepted: September 27, 2021

Published: September 30, 2021

Copyright () 2021 by author(s) and Open Access Library Inc.

This work is licensed under the Creative Commons Attribution International License (CC BY 4.0).

http://creativecommons.org/licenses/by/4.0/

\section{(c) (i) Open Access}

\begin{abstract}
Language is a unique ability of human beings, and one of the standards of language is to express information clearly, but there are ambiguities in language. Ambiguity can be found on many occasions. It is not useless; on the contrary, it is interesting that it can play a positive role in helping people achieve certain value-added speech and communication purposes. So sometimes people try to eliminate ambiguities, but sometimes, language users also deliberately create ambiguities. But at the same time, ambiguous sentences not only have an impact on communication, but also bring inconvenience to computer language recognition, machine translation, human-computer dialogue and language information processing. From the language level, the reasons for the formation of ambiguity are mainly divided into 5 aspects, namely, phonetics, vocabulary, grammar, semantics and pragmatics. This article analyzes the reasons for the generation of ambiguous sentences and the classification of ambiguous sentences, and mainly discusses the syntactic generation of ambiguous sentences. I hope that the in-depth discussion of ambiguity in this article will deepen the understanding of ambiguity.
\end{abstract}

\section{Subject Areas}

Linguistics

\section{Keywords}

Ambiguity, Syntax, Generation

\section{1. 引言}

人类因为有了语言而区别于其他动物, 语言的作用是为了交流, 所以语 言的标准中就包括清晰明白这样一条。任何一种语言的组织形式都是结构形 式和语义形式的统一, 但是在自然语言中, 歧义却长期且普遍存在, 歧义使 
得句子的意思不确定, 成了语言交流中的障碍。英国著名的语言学家 William Empson 就曾说过: “Ambiguity is the enemy we have to watch。” 因此歧义 自古就是人们研究的对象, 亚里士多德、荀子等都曾关注过歧义现象。

歧义现象并非偶然的、个别的现象, 而由于汉语自身缺乏严格的形态变 化, 就更集中体现了语言的形式和意义之间的矛盾, 语法形式和语法意义之 间的对应关系错综复杂, 歧义现象比形态发达的语言更加普遍 [1]。

汉语的结构形式和语义形式间的复杂关系, 使得汉语歧义现象在语言系 统中也更具普遍性, 所以把深层结构为关键的句法语义歧义研究作为抓手更 能揭示同形结构下汉语的深入语义关系。特别是从科技发展的要求来看, 要 研究自然语言的自动化处理, 如机器翻译、自然语言理解、人机对话等更需 要输入理解歧义现象, 这就确定了汉语句法语义歧义研究在研究中重要地位。

\section{2. 文献综述}

据记载, 柏拉图算是第一个进行歧义研究的哲学家, 他人为歧义看是论 辩和错误推理的主要原因。在柏拉图之后, 他的学生亚里士多德对歧义进行 了更全面的研究，他认为导致歧义产生的原因有 3 个: 同音字(homonymy)、 多义字(amphiboly)以及形式和表达(form and expression)。

到了索绪尔的现代语言学出现, 对语言的描写研究开始兴起。因为解释 任何的语言现象都必须进行描写, 歧义研究成了描写语言学家热衷研究的方 向。朱德熙曾说: “一种语言语法系统里的错综复杂和精细微妙之处往往在 歧义现象中得到反映。因此分析歧义现象会给我们许多有益的启示, 使我们 对于语法现象的观察和分析更加深入。” [2]乔姆斯基也曾说: “一种语法理 论, 对于所描述的语言是否适当、充分, 就看它解释歧义现象的能力。” [3] 乔姆斯基希望建立一种比传统语法更具解释力的理论系统。对歧义问题的深 入研究, 已成了事实上转换生成语法学框架的重要支柱。

作为率先对汉语的歧义进行研究的赵元任, 从英语歧义的著名例子: “The chicken is ready to eat。”引向了汉语的歧义研究, 在 《Ambiguity in Chinese》 中提出了最早的歧义结构的例子: “鸡不吃了” 与英语的 “The chicken is ready to ea.”一样。分析 “鸡” 是施事者还是受事者 [4]。而在国内, 最早研究汉语 歧义的。是朱德熙。他在 1962 年《论句法结构》里, 使用层次分析法对因层 次不同而引起的歧义进行了探讨 [5]。比如: “咬死/猎人的狗” 。动宾结构和 偏正结构的 “咬死猎人的/狗”。朱德熙、吕叔湘在《语法修辞讲话》第五讲 中有关于歧义的专门论述, 他们认为 “歧义就是一句话有两种讲法” , 会导 致 “说话不明白, 要读者伤脑筋” [6]。同时还初步给歧义分类。从七十年代 末开始, 语言学界普遍重视汉语的歧义现象。其中以徐仲华《汉语书面语言 歧义现象举例》的发表为标志, 开始了广泛研究歧义现象。

从七十年代后期到八十年代初, 吴葆棠《现代汉语词组歧义现象初探》 是我国第一篇对汉语歧义研究的专文[7]。郡敬敏、黄国营、徐仲华等研究者 也将汉语语法研究的主要方向转向了对于汉语歧义的研究, 归纳分析了汉语 歧义的格式和例子。关于汉语歧义研究的基本体系就至此形成了基本框架。 从综合文献来看, 研究汉语歧义的成果主要集中于语法、语义、语用三个层 
面。其中研究成果最丰富的是歧义的语法层面, 但是语义和语用歧义的研究 尚不足够, 相关成果为数不多 [8] [9]。

从八十年代后期到九十年代中后期, 柳广民、李峰等认为, 歧义主要分 为三个不同层次: 即语法、语义和语用的歧义。它们分别对应语言的三个不 同平面: 语法、语义和语用平面。区分研究 “歧义” 的这三个平面, 能有助 于正确地分析确定歧义语言片段的结构层次、歧义的语义内容、歧义的语用 意义[10]。

到了本世纪, 歧义研究出现了新的研究视角和领域。邱振强、迟宇风、 张炜、蔡国妹等有的研究语义结构歧义, 也就是语义成分功能相同、层次切 分相同、句法结构关系相同而语义结构关系不同的歧义样式。有的分析歧义 结构的类型, 主要把歧义结构分为语法组合歧义和语义组合歧义两大类, 以 及若干小类。

从时间阶段来说, 对汉语的歧义研究大致如上, 而从研究内容角度来说, 大概分为:

\section{1. 歧义的界定}

虽然歧义的界定研究是现代汉语研究中的一大热点, 但是专门撰文研究 歧义的界定问题的文章少之又少。第一个系统研究现代汉语歧义的是赵元任, 他给歧义的定义是 “歧义指一个符号可以有多种理解这种性质, 它跟模糊和 笼统不同。” 以后大家基本沿着这一思路, 认为歧义就是同一语言形式能同 时表达几种不同意义的现象, 只是在概念的内涵和外延上有些差异。除此而 外，各家在歧义与多义、歧义与模糊、歧义与笼统等问题上有不同的判断。

\subsection{1. 歧义与多义}

学界对 “歧义” 和 “多义” 是否一致颇有争议, 目前对 “歧义” 和 “多 义”之间关系的观点大致分为:

1) “歧义” 即 “多义”。以朱德熙、胡裕树、黄伯荣和廖序东等人为代 表。赵元任曾将词、词组和句子的多义都称为歧义。朱德熙认为 “所谓语法 歧义指的是句子的多义现象。一个词不止一个意思叫多义词, 那么一个句子 不止一个意思叫做 “多义句”。多义句有两种: 一种是句子里某个词是多义 词, 因此句子相应地就成了多义句。另一种是语法上的多义句” [11]。胡裕树 认为 “形式上是一个词组, 实际上是两个或两个以上不同类型的词组交叉在 一起, 这个词组就是多义的”。同时 “词与词之间的结关系不同, 由于词组 内部的结构层次不同, 因此产生了多义现象” [12]。黄伯荣和廖序东也认为“一 个短语同时有两种或多种意义, 这是同形异义, 叫做多义短语” [13]。

2) “歧义” 不等于 “多义”。以王维成、黄德玉等人为代表。王维成认 为 “多义是从静态的描写上去看的, 如词典中的多义词; 而歧义是从动态的 角度上去看的。歧义之所以成为歧义就是因为人们在动态理解上发生了分歧, 而对一个存在于词典中的孤立的多义词来说, 谁也不会发生理解上的分歧, 因此, 除静态的多义词以外, 无论是句法结构体还是话语片段所产生的歧义 理解都叫做歧义” 王维成指出多义是从静态的角度去看, 而歧义是因为在动 
态的理解上发生了分歧, 所以谁也不会对一个存在于词典中的多义词发生理 解上的分歧 [14]。黄德玉认为 “多义是语义平面上的语言现象”, 即作为静态 的语言单位的多义现象。“歧义是语句平面的语言现象”, 即语言单位在运 用过程中产生的歧义[15]。

3）“歧义” 和 “多义” 没有严格的区分。以邵敬敏为代表。邵敬敏认为 “多义和歧义没有严格的区分, 习惯上多义往往指词的多义现象, 如多义词。 歧义多指语法结构(词组或句子)上的多义现象, 如歧义结构”。也就是说, 无论是词的多义还是结构上的多义, 统统可以称为多义。但词的多义现象不 能称为歧义[16]。

多义和歧义都是指在同一语言表达形式的情况下有不止一个意义的现 象, 这二者既有共同之处, 也存在区别。1) 多义是静态的, 歧义是动态的。 许多学者认为, 多义是从静态的描写上去看的, 而歧义则是从动态的理解上 去看的。歧义之所以成为歧义就是因为人们在动态的理解上出现了分歧, 在 使用语言材料时产生了问题。多义是针对 “词语” 或 “短语” 来说的, 而歧 义是针对 “结构” 来说的。多义是静态的, 是属于语言的语义层面, 是属于 语言的: 而歧义是动态的, 属于语言的语用层面, 是属于言语的。2) 多义是 固有的, 歧义是衍生的。语言具有经济性原则, 这就要求语言能用有限的材 料和模式表达无限的意义。多义性是语言单位的一种特性, 是有限的语言材 料能适应千变万化的表达需要, 符合语言的经济性原则, 所以它属于正常的 现象。而歧义是在动态的理解过程中产生的, 理解过程中对多义的制约条件, 决定了歧义是可产生与否。如果制约条件充足, 则没有歧义; 反之, 则会出 现歧义, 所以歧义是潜在的, 是衍生的。

虽然多义和歧义有区别, 但也不能割裂来看。用联系的观点来看多义和 歧义, 多义是歧义的基础, 歧义是在动态的理解过程中, 对多义缺乏必要的 制约所导致的结果, 没有多义性, 歧义就无从谈起。

\subsection{2. 歧义与模糊}

歧义与模糊也是容易混淆的一对概念, 模糊集的理论是扎德(Zadeh)提出 的, 他认为现实物质世界中所遇到的客体, 经常没有精确的界限。模糊也就 是指事物的界限不清。

赵元任在《汉语中的歧义现象》指出歧义跟模糊是不同的, 模糊指一个 符号的边界不明确的情况跟明确的情况比起来显得十分突出。石安石《语义 论》认为歧义是说同一形式有不止一个意义, 而模糊与否则是指某一个意义 的状况说的 [17]。他认为换言法或翻译法是鉴别歧义与模糊的简便方法, 歧义 通过换言或翻译可以消除, 而模糊无论通过换言还是翻译都无法消除。

比如, 红色的笔和红色的口红的简单对比:

红色的笔, 可指外面笔杆为红色的笔, 也可以指里面笔芯为红色的笔, 这里是歧义。

而红色的口红, 就是模糊, 因为这里的红色一般指的是里面的口红颜色, 而红色包括很多, 比如中国红, 深红, 桃红, 橘红, 赫红等。

模糊是语言符号所表示的客观事物或对象没有一个明确的边界或特征不 
突出, 而歧义是语言符号表示的意义有多种理解的可能, 这几种理解之间的 界限相对是很清楚的。同时, 歧义是可消除的, 模糊是不可消除的。人们可 以通过增加语境来消除歧义, 而模糊的存在并不妨碍交际, 甚至是交际所必 须的。

\subsection{3. 歧义与笼统}

歧义和笼统也是我们需要加以区别的一对容易混淆的概念, 赵元任早在 年发表的《汉语中的歧义现象》一文中论述了歧义与笼统的区别, 认为符号 的笼统指它适用于若干个事物, 人们承认这些事物有差别, 也并不忽视这些 差别, 只是这些差别在使用这个符号的语境中被认为是无足轻重的。如, 汉 语的 “人” 比英语的男人或女人笼统, 它用于不需要指出两性差别的场合。 而歧义是语言的形式和内容之间的对立矛盾, 是因为一个语言形式能够包含 两个或两个以上的命题意义和含义造成的。

笼统产生的原因是出于语言的概括性, 而歧义是因为语言的内容和形式 之间对立的矛盾而产生的, 笼统性来源于语言符号的概括性, 这是语言的本 质特征之一。世界的事物和现象是无穷无尽的。而语言手段却是有限的, 这 就要求语言必须具有概括性, 语言的概括性就决定了语言的笼统性。黄德玉 也曾举了一个生动的例子 “房子”, 来指代各地不同风格的房子, 甚至是想 象中不存在的某种房子。笼统和歧义的基本区别是：笼统是绝对的, 歧义是 相对的; 笼统的内容是不可穷尽的, 歧义的 “义” 是可穷尽的; 笼统是同质 的, 歧义是异质的。

\section{2. 歧义的类型研究}

歧义是复杂的语言现象之一, 歧义的类型研究是歧义研究中的一个重要 内容。学者们对歧义从不同的角度进行了分类。赵元任《汉语中的歧义现象》 从不同的角度对歧义进行了详细的分类。按照语境不同把歧义分为词汇歧义 和语篇歧义; 按照不同的交际意图把歧义分为有意歧义和无意歧义; 按照歧 义出现的几率把歧义分为程度高的歧义和程度低的歧义；按照是否存在不同 语言的跨越把歧义分为语内歧义和语际歧义等。吴葆棠把词组的歧义分为书 面歧义和真歧义。朱德熙《汉语句法里的歧义现象》根据歧义的形成原因分 为词汇上的多义句和语法上的多义句。语法上的多义句又根据组成成分的词 类、层次构造、显性语法关系、隐性语法关系分成四类。此外, 各研究者从 不同角度，把歧义分为合理的歧义和不合理的歧义、言内歧义和言外歧义、 词汇歧义和组合歧义、客观歧义和主观歧义、语言歧义和言语歧义、生成性 歧义和理解性歧义、积极歧义修辞歧义和消极歧义、主动歧义和被动歧义、 有标歧义和无标歧义、内部歧义和外部歧义等等。

\section{3. 歧义的化解研究}

如何化解歧义也是歧义研究的一个主要内容。歧义研究的根本目的就是 找到歧义的根源从而消除歧义, 使歧义不再阻碍人们的交际, 并能服务于人 们的交际。所以怎样化解歧义也是人们要着重研究的课题之一, 学者们从语 
言的不同角度探讨了化解歧义的方式方法。

比如朱德熙在 《汉语句法里的歧义现象》提出四种化解歧义的方法: 组 成成分的词类, 层次结构, 显性语法关系以及隐性语法关系。吴新华则在《汉 语是怎样排除结构歧义的》指出化解歧义的方法包括语音、词汇、结构本身、 语法功能、标点、语义、语境等[18]。

\section{3. 歧义句的类型}

在界定歧义类型之前, 我们需再次明确 “歧义” 的概念。

歧义一直是现代汉语研究中受到广泛关注, 但是撰专文对歧义的界定问 题进行研究的文章很少, 在近五十年的歧义研究中, 只有舒善忠《从动静关 系认识歧义》, 熊文华《语法歧义的定义问题》两篇文章专门论述了歧义及 语法歧义的定义问题。下列学者们给歧义所下的定义也具有代表性。

赵元任: 歧义指一个符号可以有多种理解这种性质。

徐仲华: 一种语言形式有时可以含有两种或两种以上的意义, 可以做两 种或两种以上的分析, 这就是所谓歧义现象 [19]。

朱德熙所谓语法歧义 (grammatical ambiguity)指的是句子的多义现象 [20]。

黄成稳: 所谓歧义, 是指某个词语或某个句子的意义不确定, 可以作这 样的理解, 也可以作那样的理解[21]。

文炼、允胎：同样的字面却隐含着不同的结构和意义[22]。

石安石: 歧义现象, 就是指有些听起来相同或看起来相同的话语, 在字 面上可以有不止一种解释[23]。

傅雨贤: 在汉语里往往存在这种情况, 一个语言片段可以作两种或多种 理解, 即它的意义不止一个, 我们就把这种现象称之为歧义句[24]。

吴英才、李裕德: 在语言交际过程中, 一个词、词组或句子, 甚至一个 语段, 有时会含有两个或两个以上的意义, 可以作两种或两种以上的理解, 这就是语言的歧义现象 [25]。

王本华: 歧义是指在某一特定的语言环境中, 一种语言形式同时表示两 种或两种以上的意义, 可以做两种或两种以上分析和理解的语言现象 [26]。

这些研究尽管对歧义的定义从不同角度进行了阐释说明, 但却仍有这样 或那样的不足。有些对歧义所包含的范围不够确定, 比如赵元任指 “一个符 号”, 徐仲华指 “一种语言形式”, 黄成稳指 “某个词语或句子”, 文炼、 允胎指 “字面” , 邵敬敏指 “某种语言形式” , 石安石指 “话语”, 傅雨贤 指 “一个语言片断” , 吴英才、李裕德指 “一个词、词组或句子, 甚至一个 语段”, 王本华指 “一种语言形式”。在这里, 为了研究的方便, 我们所讨 论的歧义, 只包括短语和句子层面, 而不包括词语, 或话语层面。

把歧义的外延扩大到短语的层面上来, 这也是出于对汉语的结构特点的 考虑。汉语短语的构造和句子基本上是一致的, 短语加上一定的语调即成为 句子, 句子去掉语调即成为短语, 所以在某种程度上, 可以说汉语的短语和 句子是一回事, 鉴于此, 后文的论述中, 不再严格区分短语和句子。依据之 二是歧义和多义的关系。多义是歧义的基础, 因而在后面的论述中, 短语的 


\section{“多义”也被我们称为歧义。}

而词汇意义的歧义，只是多义的表现，而言语意义，包括上下文和交际 情景所导致的意义, 说写者和听读者的联想意义, 言外之意, 所以词汇和言 语层面, 不在歧义句的讨论范围。

根据众多学者都对歧义的定义做出过相关表述, 本文认为歧义是在静态 的语言表达或动态的言语交际中, 词语或句子有两种或更多意义, 可出现两 种或更多理解的现象。

经过对这种现象的分析, 结合语言系统内部要素与外部环境, 现代汉语 的歧义类型可以归为五类: 语音歧义、词汇歧义、句法歧义、语义歧义和语 用歧义，本文主要讨论语法中的句法因素导致的歧义。

句法歧义，是句子因结构层次不同，或是句法成分之间的关系不同而产 生的歧义。因此句法歧义可以分为两个子类，即结构层次歧义和结构关系歧 义。结构层次歧义，是指因为句子的层次切分方式不同产生的歧义; 而结构 关系歧义，则是层次划分一致，但成分之间的关系存在差异的句子中。句法 平面上的歧义现象, 主要是由词的功能类别、词语组合时形成的结构层次和 结构关系等几方面因素形成的。

1) 词的功能类型不同引起的歧义

汉语中有一些词, 在同一上下文中, 有不同的功能, 因此产生歧义。例 如:

晚饭不热了热：动词/形容词

这篇稿子你给我看看。给: 动词/介词

这座楼房间都没锁。锁: 名词, 门锁/动词上锁

2) 词和语素同形引起的歧义

有时候, 词组或句子中, 词和语素同形, 也可能造成歧义。例如:

任/何职务任何/职务

大学/生学生

3) 词和短语同形引起的歧义

有些语言形式既可以把它作为词来理解, 也可把它作为短语来看待, 因 此在特定的句法结构中，有时也会产生歧义。例如:

我要/煎鸡蛋。(词) 我要煎/鸡蛋。(偏正短语)

这里卖/肉菜。(词) 这里卖肉/菜。(联合短语)

4) 结构层次不同引起的歧义

句子当中的词是逐层组织起来的, 其中有些词先结合, 有些词后结合, 从而形成层次。若划分层次的可能性不止一种, 就容易造成歧义。例如:

同为偏正：三个/师大的学生 三个师大/的学生

同为主谓: 他们五个/一组他们/五个一组

同为偏正: 农村/中小学教师农村中/小学教师

5) 结构关系不同引起的歧义

词与词是按照一定的结构关系组合起来的, 这种结构关系有时不止一种, 有些歧义结构, 作相同层次的划分, 但由于句法关系不同, 造成了歧义。如 常见的主谓关系与偏正关系交叉, 动宾关系与偏正关系交叉, 定中式偏正关 
系和状中式偏正关系交叉等。例如:

出租汽车 动宾/偏正

我们老师同位/偏正

学生家长联合/偏正

翻译小说 动宾/偏正

6) 结构层次和结构关系都不同

有时候有歧义的短语或句子的结构层次和结构关系都不相同。在汉语里, 词与词按照线形序列组合成短语和句子, 因此, 对这些语言单位作层次和结 构分析, 可能有多种结果, 即层次和结构不同, 可能产生多种意义。例如:

咬死/猎人的狗

准备了两年/的粮食

影响了/大家的计划

你和/乔林的孩子

7) 复句的不同切分

紧缩复句有时候也存在两种切分情况, 引起歧义。例如:

我讲不过/他也得讲(假设关系) 我讲不过他/也得讲。(转折关系)
咬死猎人/的狗

准备了/两年的粮食

影响了大家/的计划

你和乔林/的孩子

\section{4. 歧义的化解及存在意义}

汉语的形式和语义的特殊性决定了汉语本身就具有很强的消除歧义的能 力。许多语言学家也都积极探索各种消歧手段, 总结起来可以概括为利用语 音手段(轻声、重音、停顿、声调等), 词汇手段(同义词替换, 添加某些虚词、 增补或省略词等), 和语法手段(换位、同义句式的变换等), 另外还可以利用 语境手段(上下文、场境、情境、文化背景等)。这些办法中, 语境手段是最 为有效也利用频率高的分化、消歧手段, 而语境分化消歧还主要针对的是书 面语歧义。

歧义受到关注的最早原因是将其作为消极的、不合规范的语言现象提出 来的, 以此尽量减少和避免歧义, 使语言表达更加精确。随着歧义研究的发 展, 研究的重点落在了语言的形式和意义之间的关系上, 以揭示同一形式如 何产生多种意义。如果语言表达形式和意义之间出现了一对多的情况时, 就 会可能出现歧义。但在文学创作或日常会话中, 人们却为了追求某种艺术效 果, 故意制造歧义, 来增强文章、会话的表达效果。

在语言实践中, 歧义往往可以作为一种特别的手段, 广泛地运用于幽默、 广告、新闻标题、日常交际、文学作品等诸多不同语言场合, 或表达复杂的 思想, 实现语言意义的增加, 突出语言的表现力, 或用以表达某种特殊的语 用效果或语用目的, 充分展示出歧义在语言使用中的交际价值、美学价值、 艺术价值等各种的语用价值。

对歧义句的研究, 不但对语言学习有指导作用, 对现在和未来越来越多 的自然语言的自动化处理, 如机器翻译、自然语言理解、人机对话等更有借 鉴意义。因为在客观世界的语言使用中, 虽然歧义大量存在, 但是人们可以 通过语境, 通过各种约束手段来化解歧义, 或者人们保持歧义来实现一定的 语义增值, 但是对于计算机的语言处理, 却缺乏这样的环境和手段, 只能一 
对一的处理。

本文在较为全面的文献基础上，对歧义句的界定、分类和化解进行了研 究。从歧义与多义、笼统、模糊等方面的区别和联系进一步明确了歧义的研 究范围介绍了歧义的分类、造成歧义的种原因, 通过对歧义类型、成因和化 解方法的分析, 进一步论证了歧义的使用价值。阐释了对歧义句研究的重要 性, 为探索语言的本质属性和语言学研究提供实际的和理性的依据和参考。

\section{基金项目}

本文系 2020 年西南民族大学中央高校基本科研业务费专项资金项目资 助(项目编号：2020SYB10)。

\section{Conflicts of Interest}

The author declares no conflicts of interest.

\section{References}

[1] 刘俊. “ N + V + 不 + A”结构的歧义探析 [J]. 社科纵横, 2008(1): 279-280.

[2] 朱德熙. 句法结构 [J]. 中国语文, 1962(8/9): 54-61.

[3] 诺姆.乔姆斯基. 句法结构 [M]. 邢公畹, 等, 译. 北京: 中国社会科学出版社, 1979.

[4] 赵元任. 汉语中的歧义现象 [C]//袁硫林, 译. 赵元任语言学论文集. 北京: 商务 印书馆, 2002.

[5]＼cjkstart朱德熙. 论句法结构[J]. 中国语文, 1962(8-9): 54-61

[6] 吕叔湘, 朱德熙. 语法修辞讲话[M]. 北京: 中国青年出版社, 1979.

[7] 吴葆棠. 现代汉语词组歧义现象初探 [J]. 延边大学学报, 1979(1): 73-90.

[8] 邵敬敏. 歧义分化方法探讨[J]. 语言教学与研究, 1991(1): 38-50+37.

[9] 黄国营. 现代汉语的歧义短语[J]. 语言研究, 1985(1): 69-89.

[10] 柳广民. 歧义类型研究[J]. 广西社会科学, 1994(6): 92-98.

[11] 朱德熙. 汉语句法中的歧义现象 [M]//现代汉语语法研究. 北京: 商务印书馆, 1980.

[12] 胡裕树, 编. 现代汉语 [M]. 上海: 上海教育出版社, 1991.

[13] 黄伯荣, 廖序东. 现代汉语[M]. 北京: 高等教育出版社, 1997.

[14] 王维成. 从歧义看句法、语义、语用之间的关系[J]. 语言教学与研究, 1988(1): 32-41.

[15] 黄德玉. 多义·模糊.笼统[J]. 安庆师范学院学报, 1988(3): 46-52.

[16] 朱一之, 王正刚. 现代汉语语法研究的现状和回顾[M]. 北京: 语文出版社, 1987.

[17] 石安石. 语义论[M]. 北京: 商务印书馆, 1993.

[18] 吴新华. 汉语是怎样排除结构歧义的[J]. 南京师范大学学报, 1984(4): 27-34.

[19] 徐仲华. 汉语书面语言歧义现象举例 [J]. 中国语文, 1979(5): 339-343.

[20] 朱德熙. 现代汉语语法研究 [M]. 北京: 商务印书馆, 1980.

[21] 黄成稳. 说歧义[J]. 中学语文教学北京, 1982(8): 32-37.

[22] 文炼, 允贻. 歧义问题 [M]. 哈尔滨: 黑龙江人民出版社, 1985.

[23] 石安石. 歧义现象种种[M]//语义研究. 北京: 语文出版社, 1994. 
[24] 傅雨贤. 现代汉语语法学(增订本) [M]. 广州: 广东高等教育出版社, 1994.

[25] 吴英才，李裕德. 现代汉语的歧义[M]. 银川: 宁夏人民出版社, 1997.

[26] 王本华. 修辞歧义说略[J]. 首都师大学报, 1993(3): 32-38.

\section{Appendix (Abstract and Keywords in Chinese) 浅析汉语歧义句的句法结构}

摘要: 语言是人类特有的能力, 而语言的标准之一就是表达信息的清晰明白, 但在语言中却存在着歧义的现象。歧义现象广泛存在, 这并不是一无用处, 相反有趣的是, 它可以发挥积极作用, 帮助人们实现一定的言语增值和交际 目的。所以有的时候人们努力的消除歧义，但有的时候，表达者也有意创造 歧义。但同时, 歧义句不仅对交流产生了影响, 也对计算机语言识别、机器 翻译、人机对话和语言文字的信息处理带来了不便。从语言层面上来说, 歧 义形成的原因, 主要分为语音、词汇、语法、语义和语用这几个方面，本文 分析了歧义句产生的原因和歧义句的分类，主要讨论歧义句的句法生成。希 望本文歧义问题探讨的深入，能加深歧义现象的理解。

关键词: 歧义, 句法, 生成 